\title{
Superfluid qubits in ultracold atom systems
}

\author{
Suzanne McEndoo
}

Physics Department, UCC

If there's one thing that makes for an exciting subject, it's the physics of extremes. Contemplating extreme speeds brought us revolutionary theory of special relativity. There is also a whole world of strange effects on the other end of the spectrum, the realm of the extremely slow and the extremely cold.

As you go from steam to water to ice, the temperature of $\mathrm{H}_{2} \mathrm{O}$ goes down but at the same time the atoms are moving more slowly. Physically, temperature is just another way of talking about the average speed of atoms. Because there is a slowest possible speed, no speed, there is a corresponding lowest possible temperature, which we call absolute zero.

\section{Making Atoms Ultra-Cold}

Ice, we all learn in school, freezes at $0^{\circ}$ Celsius. Going further down the temperature scale, nitrogen in the air will liquefy at $-196^{\circ}$ Celsius, the temperature of outer space is $-270^{\circ}$ Celsius, and absolute zero itself is $-273.15^{\circ}$ Celsius. In order to cool atoms down to such low temperatures, lasers are used to bounce photons (particles of light) off an atom, slowing its movement. This is just like trying to walk against a strong wind. Physicsts in Tyndal National Institute use lasers in this way to cool atoms to 0.00001 degrees above absolute zero.

If atoms get even closer to absolute zero, within a billionth of a degree, their behaviour changes completely. No longer are they whizzing around in different directions like in a gas, now they start to move like an army, marching along as if they were a single giant atom. This is not a solid, liquid or gas, but a new type of matter called a Bose-Einstein condensate. This is something Einstein and an Indian physicist called Bose predicted using mathematics in 1924. It took scientists another 70 years before they were finally able to create this in the lab in 1995, earning a Nobel prize for their efforts.

\section{Quantum Whirlpools}

One of the ways in which a Bose-Einstein condensate is different from liquid or gas is that it's a superfluid, so it flows without friction. Without friction, the usual rules of flow take on new forms. Once you set a superfluid flowing, it will keep moving without any outside help. If you stir a cup of regular coffee and make a whirlpool, as soon as you take the 


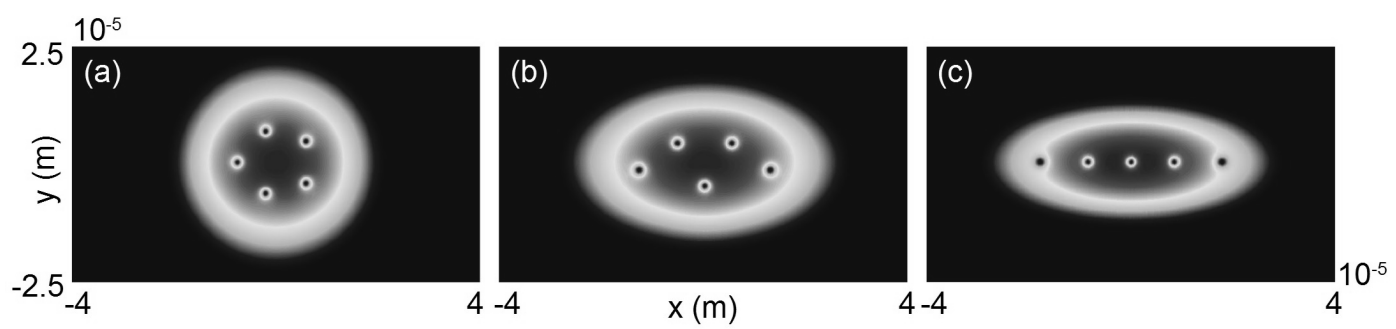

(red) with 5 vortices (blue) in a) an isotropic or round trap, b) a trap compressed 1.5 times more in the vertical than the horizontal, and c) a trap compressed twice as much in the vertical. We can see how by changing the shape of the trap containing the condensate in a very simple way, the behaviour of the vortices can be engineered. For condensates compressed by a factor of two or larger (c), the vortices form a straight line, the so-called linear vortex crystal.

Figure 1: Numerical simulations of a Bose-Einstein condensate

spoon out, friction will slow down the coffee and your whirlpool will disappear. If you instead had some superfluid coffee, that little whirlpool would stay in your mug forever. These whirlpools, or superfluid vortices, have been created in labs around the world.With a large number of these vortices in a round, or isotropic, trap, the vortices form a regular, triangular lattice. While these triangular lattices are interesting objects of study, they must generally be treated as a large object, and the motion of each individual vortex cannot be resolved.

In my research, we restrict ourselves to smaller numbers of vortices. Here, instead of a triangular lattice, rings and clusters are found in isotropic traps. We used both numerical simulations and mathematics to model these systems. We found that by simply compressing the Bose-Einstein condensate along one direction, the vortices would move from a ring to a straight line. Figure 1 shows numerical simulations of this effect for five vortices. In the first condensate, the trap is isotropic and thus a ring is formed. As the trap is compressed 1.5 times tighter in one direction, the ring structure is deformed but not fully destroyed. However, for as low as twice as compressed, the ring structure is entirely overcome and replaced by a linear vortex crystal.

\section{Quantum Information and Schrödinger's Cat}

While new forms of matter and superfluid vortices are interesting to scientists in their own right, they also have potential applications in the world of quantum information. When a physicist talks ofinformation in a computer, they talk of the physical aspects that represent the 1's and 0's of binary, current flowing or not flowing, lights on or off. Any system that can have two distinct states can encode this type of information with varying degrees of usefulness. We can even apply this to our superfluid whirlpools, with flow going clockwise being a 0 and anticlockwise a 1 . Compared to the technology in the 
average family computer this is needlessly complicated. However, there is something that the quantum world can offer that current computers can't achieve.

Erwin Schrödinger, an Austrian theoretical physicist who spent his later life living in Ireland, talked about a cat in a box, rigged with a poisoned gas. He tells us that there is a $50 \%$ chance that the cathas died, but without looking into the box, we can't tell if the cat is dead or alive. He said that this cat was in a new state, one where it is both dead and alive at the same time. Because we cannot observe a cat being both dead and alive, once we open the box and have a look, we force the cat into just one of those states. This state of being dead and alive at the same time is known as a superposition state.

While this isn't true for cats or computers (and we don't suggest you try it at home) for quantum systems like superfluid whirlpools, though, this third way of being is possible. Here, instead of "dead", "alive", and "dead and alive", we have "clockwise", "anticlockwise", and "clockwise and anticlockwise at the same time". This extra state gives a whole new way of thinking about information. For example, if you search for a number in a list, you must check each number one at atime until you find the right one, which can take a long time for a long list. However, if you could create a number which was a superposition of all the numbers in the list you can look at the superposition instead.

An additional bonus provided by the superfluid vortices is that they are more stable than many quantum systems. This is due to the fact that the fluid flow holding the information will not dissipate as a regular fluid would (like your coffee).

Through our research into physical systems, such as superfluid vortices, we can show how to engineer new and novel systems and implement this new way of computing. This global effort to create new quantum information technologies will open up the concept of information, and how we can interact with it, to a whole new world.

Suzanne McEndoo is a student in the Department of Physics in the College of Science, Engineering and Food Science, under the supervision of Dr. Thomas Busch. The author would like to acknowledge funding from Science Foundation Ireland under Project No. 05/IN/1852. 